\title{
Critical Fluctuations in Superconductors and the Magnetic Field Penetration Depth
}

\author{
Igor F. Herbut ${ }^{a}$ and Zlatko Tešanović ${ }^{b}$ \\ ${ }^{a}$ Department of Physics and Astronomy, University of British Columbia, 6224 Agricultural \\ Road, \\ Vancouver B. C., Canada V6T 1 Z1 \\ ${ }^{b}$ Department of Physics and Astronomy, Johns Hopkins University, Baltimore, MD 21218, \\ USA
}

\begin{abstract}
The superconducting transition is studied within the one-loop RG in fixed dimension $D=3$ and at the critical point. A tricritical behavior is found and, for $\kappa>\kappa_{c}$, an attractive charged fixed point, distinct from that of a neutral superfluid. The critical exponents of the continuous transition are evaluated and it is shown that the anomalous dimension of the gauge-field equals unity. This implies the proportionality of the magnetic field penetration depth and the superconducting correlation length below the transition. The penetration depth exponent is non-classical. We argue that it cannot be extracted from the dual theory in a straigthforward manner since it is not renormalized by fluctuations of the dual field.
\end{abstract}

PACS: $74.40 .+\mathrm{k}$ 
The problem of a charged scalar field coupled to a gauge vector potential arises frequently in theoretical physics. In its original version, it describes formation of Meissner state in superconductors [1] and Higgs mechanism in particle physics [2]. Furthermore, the nematicsmectic-A transition in liquid crystals [3] and, more recently, the transitions between plateaus in the quantum Hall effect [4, 5] and the finite-magnetic-field critical behavior in extreme type-II superconductors [6] have also been related to this problem. In a superconductor, the scalar field represents the fluctuating superconducting order parameter $\Psi$ which, BCS pairs being charged, is coupled to fluctuations in the electromagnetic potential $\vec{A}$. At the mean-field level the transition is discontinuous and remains so when fluctuations in $\Psi$ are included via the $\epsilon(\equiv 4-D)$-expansion [1]. Numerical simulations [7, [ of related lattice models support this scenario for smaller values of the Ginzburg parameter $\kappa$. However, for large $\kappa$, the results are consistent with a continuous, second-order phase transition [0, 8]. The picture obtained in numerical work is in accordance with the $1 / n$-expansion [1, 9] and with duality arguments which connect the lattice version of the theory to a dual gas of interacting vortex loops and the "inverted" 3D XY model [0, 10].

In this Letter we study this superconducting-Higgs electrodynamics (SHE) directly in $D=3$ within perturbation theory at the critical point corresponding to the charged superfluid. Our results are as follows: We first show that the anomalous dimension of the gauge field, $\eta_{A}$, equals unity to all orders in perturbative expansion. By combining this result with the Josephson relation, we argue that the magnetic field penetration depth, $\lambda$, and the superconducting correlation length, $\xi$, diverge with the same exponent $(\nu)$ as the transition is approached from below. These results should be contrasted with $\eta_{A}=0$ and $\lambda \propto \sqrt{\xi}$ at the unstable critical point for neutral superfluid. We then demonstrate that our one-loop results imply the presence of the tricritical point in SHE. For bare $\kappa<\kappa_{c}$ the renormalization group (RG) flows are unstable and the transition is likely first order while, for $\kappa>\kappa_{c}$, we find a stable fixed point indicating a continuous transition. By selecting the regularization 
which reproduces the established numerical value of $\kappa_{c}$, we evaluate $\nu$ and the anomalous dimension of $\Psi, \eta_{\Psi}$. Our results strongly suggest that the exponent for $\lambda$ is non-trivial and therefore different from the mean-field value suggested by Kleinert et al. [10 We propose that this difference has a physical origin and that this form of dual theory does not offer any simple way of determining the exponent of $\lambda$. Since the penetration depth is of direct experimental interest [11, 12, the description of the critical behavior within the original SHE theory retains its physical significance.

We are interested in the infrared behavior of the SHE:

$$
H=\int d^{D} \vec{r}\left[|(\nabla-i e \vec{A}) \Psi(\vec{r})|^{2}+\mu^{2}|\Psi(\vec{r})|^{2}+\frac{b}{2}|\Psi(\vec{r})|^{4}+\frac{1}{2}(\nabla \times \vec{A})^{2}\right]
$$

where $\mu^{2} \propto\left(T-T_{c 0}\right), T_{c 0}$ is the mean-field transition temperature, $b$ is a temperature independent constant, and $e$ is the charge of a BCS pair. For generality, we assume that the order parameter $\Psi$ has $n$ complex components and that the system is $D$-dimensional, $n=1$ and $D=3$ being eventually the case of physical interest. We chose to work in the gauge where the vector potential is purely transverse, i. e. where the bare gauge-field propagator is: $D_{i j}(\vec{q})=\left(\delta_{i j}-\hat{q}_{i} \hat{q}_{j}\right) / q^{2}$. First, let us discuss the non-perturbative results concerning the anomalous dimension of the gauge-field and the divergence of the penetration depth. The anomalous dimension of the gauge-field propagator is defined as:

$$
\eta_{A}=-\lim _{p \rightarrow 0} \frac{d \log Z_{A}}{d \log (p)}
$$

where $Z_{A}$ is the gauge-field renormalization factor and $p$ is the momentum of the gauge-field propagator. The $\beta$-function for the charge is

$$
\beta_{e}=\frac{d \hat{e}_{r}^{2}}{d \log (p)}=\hat{e}_{r}^{2}\left(D-4-\frac{d \log Z_{A}}{d \log (p)}\right)
$$

where $\hat{e}_{r}^{2}=e_{r}^{2} / p$ is the dimensionless renormalized charge. On approaching the critical point, $\hat{e}_{r} \rightarrow \hat{e}_{0}$ and $\beta_{e} \rightarrow 0$, so taking the limit $p \rightarrow 0$ in Eq. (3) we obtain:

$$
\hat{e}_{0}^{2}\left(D-4+\eta_{A}\right)=0
$$


Assuming a stable charged fixed point in the theory $\hat{e}_{0} \neq 0$ one obtains

$$
\eta_{A}=4-D
$$

This exact result has an important physical consequence, since it modifies the relation between the correlation length and the penetration depth as the critical point is approached from below. In general, the penetration depth scales with the superfluid density below the transition as:

$$
\lambda^{\eta_{A}-2} \propto \rho_{s}
$$

where $\rho_{s} \propto \xi^{2-D}$ is the Josephson relation [13]. Close to the transition controlled by the attractive charged fixed point it follows that

$$
\lambda \propto \xi
$$

for all $D$. In contrast to the scaling governed by the XY fixed point, where $\eta_{A}=0$ and $\lambda \propto \xi^{(D-2) / 2}$, the ratio between the two lengths close to the charged critical point approaches a finite constant. The divergences of both lengths are determined by the same exponent $\nu$.

To obtain the flow-diagram for the coupling constants and the values of critical exponents one must rely on some approximation for the $\beta$-functions. Here we perform the perturbative calculation of $\beta$-functions in fixed dimension and at the critical point. To the lowest order in perturbation theory, the contributions to the self-energy, polarization and the quartic vertex are given by the diagrams in Fig. 1. Due to the choice of gauge, the remaining one-loop diagrams for the quartic vertex all vanish when the external momenta go to zero [2]. Note that this procedure explicitly preserves Ward identities associated with gauge invariance. Since we wish to work directly in $D=3$, we are forced to define the renormalized value of $b$ at a finite momenta of external legs to avoid the infrared divergence in the last diagram on Fig. 1. This divergence is a consequence of gauge invariance which requires massless gauge-field. The renormalized coupling constant $b_{r}$ is defined at the usual 
symmetric point:

$$
\vec{k}_{i} \cdot \vec{k}_{j}=\frac{4 \delta_{i j}-1}{4} p^{2}, \quad i, j=1,2,3
$$

and at the critical point where the renormalized mass of $\Psi$ vanishes. There are two relevant coupling constants in the problem: the quartic term coupling and the charge. The standard procedure [14] gives the renormalized coupling constants to lowest order:

$$
\begin{gathered}
e_{r}^{2}=e^{2}+\frac{2 n \Gamma(1-D / 2) \Gamma^{2}(D / 2)}{(4 \pi)^{D / 2} \Gamma(D)} e^{4} q^{D-4} \\
b_{r}=b-\left(\frac{n+3}{2^{2-D / 2}}+1\right) \frac{\Gamma(2-D / 2) \Gamma^{2}(D / 2-1)}{(4 \pi)^{D / 2} \Gamma(D-2)} b^{2} p^{D-4}+ \\
\frac{4(D-1) \Gamma(2-D / 2) \Gamma(D / 2-1) \Gamma(D / 2)}{(4 \pi)^{D / 2} \Gamma(D-1)} b e^{2} p^{D-4}-\frac{2(D-1) \Gamma(2-D / 2) \Gamma^{2}(D / 2-1)}{(4 \pi)^{D / 2} \Gamma(D-2) 2^{2-D / 2}} e^{4} p^{D-4},
\end{gathered}
$$

where $\Gamma(x)$ is the factorial function, the momentum scale $p$ is defined above and $q$ is the momentum of the gauge-field propagator. Hereafter, we set $D=3$ and $n=1$ and define dimensionless couplings with respect to the external momentum $p: \hat{b}=b / p, \hat{e}^{2}=e^{2} / p$. If we choose the reference momentum of the gauge-field propagator as $q=p / c$, where $\mathrm{c}$ is a constant, the $\beta$-functions are:

$$
\begin{gathered}
\beta_{e}(\hat{b}, \hat{e}) \equiv \frac{d \hat{e}^{2}}{d \log (p)}=-\hat{e}^{2}+\frac{c}{16} \hat{e}^{4} \\
\beta_{b}(\hat{b}, \hat{e}) \equiv \frac{d \hat{b}}{d \log (p)}=-\hat{b}+\frac{1}{8}(2 \sqrt{2}+1) \hat{b}^{2}-\frac{1}{2} \hat{b} \hat{e}^{2}+\frac{1}{2 \sqrt{2}} \hat{e}^{4},
\end{gathered}
$$

and we dropped the subscript " $r$ " for renormalized coupling constants in the last two equations.

The above $\beta$-functions explicitly depend on the introduced ratio of momenta, $c$. This reflects the known property of the $\mathrm{RG}$ in fixed dimension that, unlike in the $\epsilon$-expansion scheme, the coefficients in the perturbative series for $\beta$-functions are procedure dependent [14]. Consequently, an additional information is needed to fix the value of $c$ in the one-loop calculation. We now note an important property of Eqs. (10,11): If $c$ is treated as a free 
parameter, the RG flow diagram has a structure pictured in Fig. 2 (for $c>5.16$ ). Besides the standard Gaussian and the neutral superfluid fixed points, there are two charged fixed points of the above flow equations: $\left(\hat{b}_{-}, \hat{e}_{0}^{2}\right)$ and $\left(\hat{b}_{+}, \hat{e}_{0}^{2}\right)$, where $\hat{e}_{0}^{2}=16 / c$ and $\hat{b}_{+}$and $\hat{b}_{-}$ are the real roots of the equation $\beta_{b}\left(\hat{b}, \hat{e}_{0}\right)=0$. Stability analysis shows that the fixed point with the larger value of $\hat{b}=\hat{b}_{+}$is attractive, while the one with $\hat{b}=\hat{b}_{-}$is unstable in the direction of quartic term coupling. Now we look for the straight RG trajectories, $\hat{b}=2 \kappa^{2} \hat{e}^{2}$, by demanding the invariance of the constant $\kappa$ under RG transformation:

$$
\frac{d\left(\hat{b} / \hat{e}^{2}\right)}{d \log (p)}=0
$$

In our one-loop analysis this leads to the equation:

$$
\beta_{b}\left(2 \kappa^{2} \hat{e}_{0}^{2}, \hat{e}_{0}^{2}\right)=0
$$

resulting in two straight-line RG trajectories for which

$$
\kappa_{+,-}^{2}=\frac{\hat{b}_{+,-}}{2 \hat{e}_{0}^{2}}
$$

Together with the result for the stability of the $\left(\hat{b}_{-}, \hat{e}_{0}^{2}\right)$ fixed point, this implies that there is a tricritical line in the theory given by a GL parameter:

$$
\kappa_{c}^{2}=\frac{\hat{b}_{-}}{2 \hat{e}_{0}^{2}}=\frac{c+8-\sqrt{c^{2}+16 c-32(2+\sqrt{2})}}{8(2 \sqrt{2}+1)} .
$$

This solution appears physically plausible and is in accord with Ref. [15] in that the tricritical point is determined by a particular value of the Ginzburg parameter, as one would expect from a mean-field argument. Consequently, we fix the value of $c$ by demanding that $\kappa_{c}$ in Eq. (15) matches the value obtained via the duality transformation of the lattice SHE [15] (see also numerical Monte Carlo results of Ref. [8]): $\kappa_{c} \cong 0.8 / \sqrt{2}$. This condition results in $c=5.7$ (> 5.16). The other straight trajectory given by the $\kappa_{+}$solution connects the Gaussian and the superconducting fixed point. 
Having fixed the value of the parameter $c$ we thus obtain the flow diagram of Fig. 2 [16], [17]. The exponents at the attractive fixed point are

$$
\begin{gathered}
\eta_{\Psi}=\frac{-\hat{e}_{0}^{2}}{4}=-0.70, \\
\nu=\frac{1}{2}\left(1+\frac{\hat{b}_{+}}{8}-\frac{\hat{e}_{0}^{2}}{8}\right)=0.53,
\end{gathered}
$$

with the numerical values calculated for $c=5.7,\left(\kappa_{c}=0.8 / \sqrt{2}\right)$. It is worth mentioning that our procedure gives a respectable value for the correlation length exponent at the neutral superfluid fixed point: $\nu_{X Y}=0.63$. The value of the anomalous dimension is rather large and negative but it does satisfy $\eta_{\Psi}>-1$ in $D=3$. Note that small reductions in the assumed value for $\kappa_{c}$ rapidly make $\eta_{\Psi}$ less negative (as do the next order perturbative terms) while the value for $\nu$ is more robust. For example, $\kappa_{c}=0.45 / \sqrt{2}$ [ 8 gives $\eta_{\Psi}=-0.20$ and $\nu=0.62$. The other exponents follow from standard scaling relations. It is conceivable, however, that the hyperscaling relation does not hold, due to the presence of long-range gauge forces in (1). In that case we could define a characteristic dimension, $d_{c}$, from $2-\alpha=d_{c} \nu$. By combining our results with the prediction of the dual theory $\alpha=\alpha_{X Y}=-0.013$ one obtains $d_{c} \cong 3.8$. This is close to $d_{c}=4$ which would arise from a naive scaling of current-current interactions.

To the lowest order in $\epsilon \equiv 4-D$ the $\beta$-functions derived from Eqs. (9) completely reproduce the results of the RG defined at zero external momenta and finite mass [1, 16]. This is to be expected since the dimensional regularization and the minimal subtraction scheme lead to unique values of the coefficients in the $\beta$-functions [14]. The parameter $c$ then does not appear at all, the attractive fixed point exists only for $n>182.95$ and the exponents agree with ours in this limit. 18

The exponent for the magnetic field penetration depth is $\nu=0.53(0.62)$. While this appears close to the mean field value of $1 / 2$ suggested by Kleinert et al. [10] on the basis of the dual theory, it is clear from our procedure that this exponent is non-trivial and determined by the structure of the charged fixed point. This is an important aspect of the physics of this 
problem: In the dual approach the partition function of the original problem is related to the one for the interacting vortex loops [7]. The dual description is a theory of a scalar complex field coupled to a massive vector potential [10] and is in the universality class of the 3D XY model [10]. This description could be useful in providing the information on thermodynamic quantities. For example, the specific heat exponent $\alpha$ can be calculated from the hyperscaling relation, which holds in the dual theory, and has the value for the 3D XY model. The dual approach, however, offers little help in calculating exponents that characterize correlation functions of the original superconducting order parameter. In particular, the magnetic field penetration depth appears in the dual description via the mass of the "dual" vector field. If one assumes that this mass vanishes fast enough at the transition at the bare level, then, at the critical point of the dual theory, this mass does not renormalize, i.e. it remains equal to its bare value. [19] Since the mass of the dual vector field is what determines the inverse of the penetration depth, the implication is that the divergence of the penetration depth at the superconducting transition cannot be extracted from the RG analysis of the dual model, even in principle. To calculate the penetration depth exponent it is necessary to apply the RG analysis to the original SHE theory. This fact supplied the primary motivation for the present work.

Our value for the penetration depth exponent, $\nu=0.53$, is not seen in the experimental results of Ref. [11], which are consistent with the neutral 3D XY behavior, but is in excellent agreement with those of Ref. [12]. While the critical region in which charge of BCS pairs is relevant is rather narrow (even for HTS) due to the small value of the fine structure constant, the fact that our exponent is quite close to the mean-field value may effectively "enlarge" this region. In general, as we approach the transition from below, we expect that the meanfield behavior of the penetration depth crosses over to the intermediate regime of neutral superfluid followed by the ultimate charged superfluid critical behavior. The exponent in this intermediate $3 \mathrm{D} \mathrm{XY}$ regime is $\sim 1 / 3$, considerably different from both the mean-field 
value and the ultimate value of $\sim 0.53$. Consequently, on purely empirical grounds, the region over which the penetration depth can be described by the crossover behavior should be quite narrow. Finally, we should mention that experiments on other systems [4, 5, 6] might be even more promising in studying the SHE critical behavior since there the effective "fine structure constant" can be of order unity.

In summary, we studied the superconducting transition by calculating the one-loop $\beta$ functions in fixed dimension $D=3$. The gauge invariance leads to infrared divergences which are handled by defining the renormalized coupling constants at finite values of the external momenta and right at the critical point. We eliminate the remaining freedom in the definition of renormalized couplings by requiring that our analysis yields to the previously established [15, 8] numerical value of the Ginzburg parameter which characterizes the tricritical point. We then evaluate critical exponents at the attractive charged fixed point of our theory. It is shown that the penetration depth and the correlation length diverge in the same way close to the charged fixed point, to all orders in perturbation theory. [20]

The authors acknowledge useful conversations with Professors I. Affleck and O. T. Valls and hospitality of the Aspen Center for Physics where part of the work was performed. This work has been supported in part by the NSF Grant No. DMR-9415549. IFH has also been supported by NSERC of Canada. 


\section{Figure Captions:}

Figure 1. Lowest order contributions to the self-energy (a), polarization (b) and the quartic vertex (c). The full and dashed lines are the order parameter and the gauge-field propagators, respectively.

Figure 2. The schematic flow-diagram for the dimensionless charge $\hat{e}$ and the dimen-

sionless quartic term coupling $\hat{b}$ (for $c>5.16$ ). Note that, for $c=5.7, \hat{e}_{0}^{2}=2.81, \hat{b}_{+}=3.22$, $\hat{b}_{-}=1.80, \kappa_{+}=1.07 / \sqrt{2}, \kappa_{-}=0.80 / \sqrt{2}$. 


\section{References}

[1] B. I. Halperin, T. C. Lubensky and S. K. Ma, Phys. Rev. Lett. 32, 292 (1974).

[2] S. Coleman and E. Weinberg, Phys. Rev. D 7, 1988 (1973).

[3] P. G. DeGennes, Solid State Commun. 10, 753 (1972); T. C. Lubensky and J-H. Chen, Phys. Rev. B 17, 366 (1978).

[4] X. G. Wen and Y. S. Wu, Phys. Rev. Lett. 70, 1501 (1993).

[5] L. Pryadko and S. C. Zhang, Phys. Rev. Lett. 73, 3282 (1994).

[6] Z. Tešanović, Phys. Rev. B 51, 16204 (1995).

[7] C. Dasgupta and B. I. Halperin, Phys. Rev. Lett. 47, 1556 (1981).

[8] J. Bartholomew, Phys. Rev. B 28, 5378 (1983).

[9] L. Radzihovsky, Europhys. Lett. 29, 227 (1995).

[10] M. Kiometzis, H. Kleinert and A. M. J. Schakel, Phys. Rev. Lett. 73, 1975 (1994); Fortschr. Phys. 43, 697 (1995).

[11] S. Kamal, D. A. Bonn, N. Goldenfeld, P. J. Hirschfeld, R. Liang, and W. N. Hardy, Phys. Rev. Lett. 73, 1845 (1994).

[12] Z. H. Lin, G. L. Spalding, A. M. Goldman, B. F. Bayman, and O. T. Valls, Europhys. Lett. 32, 573 (1995). This paper also contains a detailed comparison with Ref. [11].

[13] B. D. Josephson, Phys. Lett. 21, 608 (1969).

[14] J. Zinn-Justin, Quantum field theory and critical phenomena, (Oxford University Press, Oxford 1993); J. J. Binney, N. J. Dowrick, A. J. Fisher, and M. E. J. Newman, The theory of critical phenomena, (Oxford University Press, Oxford 1992). 
[15] H. Kleinert, Lett. Nuovo Cimento 35, 405 (1982).

[16] The flow-diagram with the same topology follows from the large- $n$ limit in $D=4-\epsilon$, see, for example: I. D. Lawrie, Nucl. Phys. B 200 (FS 14), 1 (1982); J. Phys. C: Solid State Phys. 15, L879 (1982); 16, 3527 (1983).

[17] A. Kovner, P. Kurzepa and B. Rosenstein, Mod. Phys. Lett. A 14, 1343 (1993).

[18] In this context we should note that within our theory the numerical value for $c(=5.7)$ is sufficiently large to allow for charged critical points even in $3 \mathrm{D}$ (i.e., $\epsilon=1$ ) and for $n=1$.

[19] Under the assumption that the penetration depth $\lambda$ goes as $\left(T_{c}-T\right)^{-\mu}$, our RG analysis of the dual theory shows that $\lambda$ is not renormalized by the fluctuations of the $d u a l$ field if $\mu>1 / 3$. The mean-field exponent for $\lambda$ suggested in Ref. [10 satisfies this condition (as does the value 0.53 found in this work) but it is clear from our analysis that this exponent cannot be determined solely from the dual theory. The exponent for $\lambda$ is renormalized by the critical fluctuations of the superconducting order parameter and takes on a non-trivial value when such fluctuations are accounted for within the original SHE theory.

[20] After this work was completed, the paper by B. Bergerhoff, F. Freire, D. F. Litim, S. Lola, and C. Wetterich [Phys. Rev. B 53, 5734 (1996)] has appeared in which the SHE theory is studied by applying various truncations to the non-perturbative RG flow equations. 
This figure "fig1-1.png" is available in "png" format from: http://arxiv.org/ps/cond-mat/9605185v1 
This figure "fig1-2.png" is available in "png" format from: http://arxiv.org/ps/cond-mat/9605185v1 